\title{
Civilisations
}

Revue internationale d'anthropologie et de sciences

humaines

63 | 2014

L'Ancestralité revisitée

\section{L'ancestralité revisitée}

\section{Emmanuelle Kadya Tall}

\section{OpenEdition \\ Journals}

Édition électronique

URL : http://journals.openedition.org/civilisations/3637

DOI : 10.4000/civilisations.3637

ISSN : 2032-0442

\section{Éditeur}

Institut de sociologie de l'Université Libre de Bruxelles

\section{Édition imprimée}

Date de publication : 30 septembre 2014

Pagination : 11-24

ISSN : 0009-8140

\section{Référence électronique}

Emmanuelle Kadya Tall, «L'ancestralité revisitée », Civilisations [En ligne], 63 | 2014, mis en ligne le 30 septembre 2015, consulté le 10 décembre 2020. URL : http://journals.openedition.org/civilisations/ 3637 ; DOI : https://doi.org/10.4000/civilisations.3637 


\title{
L'ancestralité revisitée
}

\author{
Emmanuelle Kadya TALL
}

Le culte des ancêtres a longtemps été un des objets d'étude favori de l'anthropologie classique, africaniste tout du moins. Puis la mode s'en est perdue, peut-être parce que les lignages, dans le cadre desquels le culte s'effectuait, ont cessé d'être perçus comme des réalités stables et perpétuelles. Dans cette désaffection s'est peut-être perdu quelque chose qui était présent chez nos prédécesseurs, à savoir que le culte des ancêtres, ou vaudrait-il mieux dire la notion d'ancestralité, comportait des aspects touchant à la constitution de la personne, à la transmission entre générations et aux rapports qu'une société entretient avec son passé. En revisitant l'ancestralité, il s'agit de montrer comment ce phénomène persiste, en dehors des agencements familiaux lignagers. La manière dont certains segments des sociétés contemporaines s'emparent du schème de l'ancestralité vient témoigner du fait que le recours aux ancêtres doit se comprendre bien plus comme un idiome que comme un modèle inhérent aux religions polythéistes en opposition aux religions universalistes. Il s'agit donc moins, dans ce numéro, de reconsidérer la littérature anthropologique classique et contemporaine sur le culte des ancêtres, que de s'interroger sur les processus d'ancestralisation de certaines figures dans un monde qui, dans un même mouvement, est à la fois, de plus en plus partagé, grâce aux nouvelles techniques d'information et divisé, en se redéployant dans une multiplicité des identités et des cultures. Le recours à des figures ancestrales participe de ces redéploiements identitaires, même si, nouveau paradoxe, ces figures tutélaires rassemblent tout autant qu'elles ne divisent, et cela bien au-delà de la sphère des agencements familiaux. Plus encore, il convient de souligner que les processus d'ancestralisation ne sont plus toujours précédés de rites mortuaires ou funéraires. Au contraire, ils adviennent souvent à la suite de rites d'installation d'abord tiraillés entre des pratiques de mémorisation, de commémoration et de patrimonialisation. Ainsi, le rite advient sans qu'il y ait eu nécessairement cérémonial mortuaire. C'est comme si le sens du rite était inversé, on identifie/fabrique un ancêtre et ensuite on lui offre des sacrifices pour établir un lien généalogique avec lui, une marque d'identification à lui. De cette manière, l'ancestralité permet de circonscrire des territoires de tout ordre : généalogique, sacré, socio-ethnique, géographique, génétique, etc.. 


\section{Le culte des ancêtres comme rite instituant}

En revisitant la littérature anthropologique classique et contemporaine, on observe deux courants, anglo-saxon et francophone, qui mettent en avant l'ordre de la parenté, cependant à partir d'approches différentes. Le premier part d'une approche juridique en interrogeant la séniorité et en ouvrant sur le rapport que la société entretient avec le temps. Le second insiste sur les funérailles.

Le courant anglo-saxon introduit la notion d'autorité juridique et politique fondée sur l'institution des secondes funérailles. Des auteurs comme Goody (1962) et Fortes (1965) rompant avec la perspective évolutionniste qui considérait le culte des ancêtres comme une métaphysique et une forme primitive de religion, ont établi l'importance des liens de consanguinité au sein des institutions (Fortes 1965 : 122). En effet, le culte des ancêtres concerne toujours un groupe de filiation et permet de distinguer ces groupes les uns des autres. Il doit également être distingué du culte des morts, distinction établie par Gluckman (1937) qui soulignait que tous les morts n'accédaient pas nécessairement au statut d'ancêtre. Fortes introduit la notion d'autorité juridique en affirmant que « c'est seulement la composante d'autorité existant dans les relations entre générations successives qui est transformée en ancestralité » (Fortes 1965 : 130). Cependant pour ces porteurs d'autorité, la mort ne suffit pas à les transformer en ancêtres car l'ancestralisation est aussi le fruit d'un processus rituel généralement réalisé dans les sociétés africaines, lors des secondes funérailles. Ainsi chez les Tallensi, l'enterrement est suivi d'une consultation divinatoire qui demande aux ancêtres s'ils acceptent que le défunt siège parmi eux. Fortes raconte qu'ayant un jour assisté à cette séance divinatoire après l'enterrement d'un homme que tout le monde estimait : l'oracle refusa l'ancestralisation en donnant pour raison que l'homme était mort d'une mort naturelle, et non pas parce que les ancêtres l'avaient rappelé à eux. Il apprit plus tard que l'homme en question était né esclave et malgré une vie respectable son statut lui déniait le droit à l'ancestralité.

On retiendra donc des travaux de Fortes que l'ancestralité concerne au moins trois pôles : l'inscription dans un groupe, la transmission de l'autorité entre générations, et la constitution de la personne morale d'un individu mais également la personne tout court, puisque la réincarnation de quelque chose d'un défunt dans un nouveau-né est une croyance qui se rencontre dans de nombreuses sociétés.

Igor Kopytoff (1971) montre, quant à lui, que la tradition anthropologique qui expliquait le culte des ancêtres en termes fonctionnalistes de modèle « juridique » de l'organisation sociale avait atteint « un cul-de-sac théorique ». Il a cherché en même temps à prolonger les travaux de Fortes en interrogeant même la notion d'ancêtre comme biais ethnocentrique introduisant de la dichotomie entre aînés et ancêtres alors qu'il y voyait, à partir d'un terrain chez les Suku du Congo Kinshasa, un continuum. Une des ambitions de Kopytoff était d'expliquer que certaines sociétés d'une même aire culturelle présentent des cultes d'ancêtres «typiques » et d'autres non, selon qu'elles accordaient une plus ou moins grande importance à l'aînesse et à la séniorité. Cet article suscitera une levée de boucliers et signera de facto, un désinvestissement des anthropologues pour la thématique pendant de longues années.

Dans les années 1990, John McCall (1995) s'interroge sur le fait que la problématique des cultes africains aux ancêtres semble avoir disparu de la production 
anthropologique. Il constate que l'article de Kopytoff qui avait pour but de revitaliser les études sur l'ancestralité lui avait au contraire donné un coup d'arrêt. Plus largement, il lie cette désaffection de l'intérêt des chercheurs au fait que les théories du lignage et de l'organisation sociale segmentaire qui sous-tendaient l'analyse des cultes aux ancêtres ont été remises en question (Kuper 1982). Les lignages étant désormais considérés « comme des constructions idéologiques dynamiques », et non plus comme des réalités stables et perpétuelles, l'étude de l'importance des ancêtres dans le domaine juridico-social a été délaissée. Mais McCall tient à souligner que les ancêtres ont un domaine d'influence beaucoup plus large, comme le rapport au passé d'une société et la constitution de la personne, invitant donc les chercheurs à s'intéresser de nouveau aux ancêtres.

En France, l'équipe du laboratoire «Système de pensée en Afrique noire » a consacré dans les années 1990 trois numéros de sa revue à la problématique de l'ancestralité à travers le deuil et ses rites ${ }^{1}$. Le point de départ de cette réflexion était, dans une perspective hertzienne (Hertz 1970 : 1-83), d'envisager ce qui dans les rites funéraires africains est conçu comme un travail de séparation du mort et des survivants, ce qui s'y montre d'une « défaite » progressive des liens qui attachent le défunt à ce monde, pour utiliser une formule de Robert Hertz. Cette étape étant le préalable à l'installation du défunt en tant qu'ancêtre.

Le regain d'intérêt pour le culte des ancêtres dans leur historicité et dans leur contemporanéité est marqué par les conflits et les tensions qui s'expriment aujourd'hui en Afrique autour des funérailles ${ }^{2}$. Dans beaucoup de sociétés, les funérailles et les commémorations des morts sont devenues les plus importants et les plus coûteux des événements auxquels les communautés familiales sont confrontées. Un aspect du changement social qui jusqu'à récemment n'a pas été analysé de manière systématique et qui n'apparaissait qu'au détour d'ouvrages consacrés à d'autres problématiques. Par exemple, le culte syncrétique du bwiti gabonais résulte de la transformation du culte des ancêtres à reliques (le crâne du défunt) que pratiquaient les Mitsogo du centre du Gabon.

Depuis les années 2000, l'intérêt des anthropologues pour le changement dans les pratiques funéraires en Afrique a été renouvelé par les travaux de Marleen de Witte (2001) au Ghana, de Joseph Tonda (2000) au Congo et plus récemment par les travaux de Michael Jindra (2005) et Joël Noret (2010) respectivement au Cameroun et au Bénin.

Les funérailles sont devenues le lieu par excellence où le pouvoir de représentation s'exerce et parfois de manière conflictuelle. La cruauté des rapports sociaux, d'âge, de classe sociale, de genre et de statut, s'y donne à lire, ainsi que les appartenances religieuses parfois antagonistes des membres de la famille du défunt : les accusations de sorcellerie étant fréquentes dans les sociétés où l'interrogation du cadavre participe du rituel funéraire, la conversion au Christianisme permet dans certains cas d'enrayer la prolifération des sorciers au profit des ancêtres, ouvrant ainsi aux jeunes et aux femmes l'accès à l'ancestralité ${ }^{3}$.

1 Voir les cahiers 9 (1989), 11 (1991) et 13 (1994) de Systèmes de Pensée en Afrique Noire.

2 Voir l'article précurseur de Claudine Vidal (1986) sur les « Funérailles et conflit social en Côte d'Ivoire ».

3 Voir les travaux menés par Michaël Jindra dans les Grassfields au Cameroun. 
On aura compris que dans les sociétés africaines précoloniales, l'ancestralité n'était pas ouverte à tous et que parmi ceux susceptibles d'être élus, la mort en soi n'était pas suffisante et qu'un processus rituel souvent long et toujours marqué par deux étapes (premières et secondes funérailles) était impératif dans la fabrication d'un ancêtre.

Or, depuis 25 ans, on assiste un peu partout dans le monde à une résurgence des ancêtres et à leur prolifération en dehors de schéma lignager et c'est à ce phénomène que ce numéro spécial prétend s'attacher.

\section{Des ancêtres à tout vent}

Le succès des recherches généalogiques qui a pris son essor dans les années 1990 ne se dément pas. Si le phénomène roots concernait dans les années 1970, principalement les descendants d'Africains Américains en quête de racines leur permettant de renouer avec l'Afrique, aujourd'hui, la quête d'ancêtres n'est plus le seul fait de diasporas issues de l'esclavage. En effet, après le retour à la terre consécutif aux mouvements de Contre-culture dans les mêmes années, mouvements réagissant à l'industrialisation et l'urbanisation intensive dans les pays du Nord et depuis le tournant du troisième millénaire, l'intérêt pour l'ancestralité accompagne dans le même temps, les progrès technologiques (les TIC et la science génomique en particulier) et les processus de mise en patrimoine de la diversité des identités et des cultures à travers le monde globalisé.

En revisitant l'ancestralité, il s'agit de questionner les processus d'ancestralisation de certaines figures dans un monde qui, dans un même mouvement, est à la fois, de plus en plus partagé grâce aux nouvelles techniques d'information et divisé, dans une refonte des identités et des cultures, participant à ce que François Hartog (2003) qualifie de présentisme, ce régime d'historicité contemporain qui configure la tension entre les pratiques mémorielles, de patrimonialisation et de commémoration.

F. Hartog définit la notion de présentisme dans le cadre des réflexions sur les régimes d'historicité. Ces derniers visent à rendre compte des façons plurielles dont les communautés humaines vivent leur rapport au temps et de la manière dont elles ont pensé les divers découpages du temps à partir d'un certain nombre d'invariants, de catégories transcendantales. Il devient ainsi possible d'en repérer les conditions pour penser les diverses modalités de division du temps dans les civilisations. Cette notion établit un pont entre la part subjective du sujet historique, individuel ou collectif, et l'état objectif d'existence dans le temps. Un régime d'historicité se définit par la forme qu'emprunte au sein d'une société l'articulation entre catégories reçues et contextes perçus, entre significations culturelles et impératifs pragmatiques, définissant chacun des configurations singulières. Cette notion élaborée aux confins de l'histoire et de la philosophie, s'est particulièrement développée à partir des années 1980 pour tenter de saisir la spécificité du temps présent au sein de la période contemporaine, au sens classique du découpage historien. Observant depuis la fin des années 1980 la fin des grands récits et la difficulté à se projeter dans le futur, Hartog constate l'apparition d'un véritable duel entre mémoire et histoire. Le patrimoine et la mémoire continuent d'être des vecteurs d'identité, cependant souligne Hartog (2003 : 165), « ... il s'agit moins d'une identité évidente et sûre d'elle-même, que d'une identité s'avouant inquiète, risquant de s'effacer ou déjà largement oubliée, oblitérée, réprimée : d'une identité à la recherche d'elle-même, à exhumer, à bricoler, voire à inventer ». 
Le présentisme s'organise autour de trois termes : mémoire, patrimoine et commémoration. On s'appuie sur la mémoire en tant qu'elle est volontaire, provoquée et reconstruite selon la méthodologie des lieux de mémoire. Le patrimoine est valorisé, promu de manière active. L'important est qu'il soit visible, investi de signification. Les commémorations rythment le calendrier de la vie publique. Elles s'adaptent à la pédagogie et au message politique du jour, selon une mise en scène symbolique.

Ces mobilisations mémorielles sont contemporaines de la chute du monde soviétique et certains textes de ce numéro présentent tant en Afrique que dans les Amériques noires, des entreprises patrimoniales mémorielles et de commémoration où l'invention et la reconstruction du passé sont mobilisées autour de la mémoire de l'esclavage, de la résistance des Noirs marrons et la reconnaissance de cultures religieuses n'appartenant pas au complexe abrahamique.

Le recours à des figures ancestrales participe de redéploiements identitaires et mémoriels, même si, nouveau paradoxe, ces figures tutélaires rassemblent tout autant qu'elles ne divisent, et cela bien au-delà de la sphère des agencements familiaux lignagers. Mais dans la fabrication d'un ancêtre, tout en nécessitant le passage de la vie à trépas pour accéder à ce statut honorifique, le processus d'ancestralisation n'est plus toujours précédé de rites mortuaires ou funéraires. Les auteurs observent ici en effet, un réinvestissement ou un investissement renouvelé pour la fabrication d'ancêtres hors du champ des funérailles. Il convient alors de noter que les processus d'ancestralisation de figures d'un passé plus ou moins lointain n'obéissent pas aux mêmes logiques de séparation, agrégation qu'on retrouve classiquement dans les rites mortuaires et funéraires. En effet, ce qui s'observe de plus en plus, c'est le surgissement d'ancêtres quasi ex nihilo, dans une manipulation/instrumentalisation très postmoderne des subjectivités de chacun.

Ainsi, le rite advient sans qu'il y ait eu nécessairement cérémonial mortuaire. C'est comme si le sens du rite était inversé, on identifie/fabrique un ancêtre et ensuite on lui offre des sacrifices pour établir un lien généalogique avec lui, une marque d'identification à lui. Les processus d'ancestralisation ne passent donc plus nécessairement par la case rite mortuaire, et les sacrifices oblatifs qui les accompagnent réunissent bien souvent une série d'acteurs/personnages/figures de nature parfois fort différente. Ce qui est, par exemple, très significatif dans le candomblé, où l'on voit célébrer ensemble, divinités de la nature, figures génériques de l'autochtonie, ancêtres de lignées symboliques et ancêtres biologiques. Néanmoins, il y a toujours des sacrifices et des processus de transsubstantiation pour que s'active le lien entre l'ancêtre et ceux qui se réclament de lui.

Dans ce numéro, il s'agit aussi de réinterroger la question de l'absence d'ancestralité aux Antilles ou en contexte amazonien en regard avec l'ancestralité inventée. En effet, il convient de rappeler que les sociétés amazoniennes se caractérisent par une faible profondeur généalogique, deux générations au plus, impliquant l'absence de lignées transmettant des droits ou des biens. Par ailleurs dans les Caraïbes et au Brésil, il y a un paradoxe à mobiliser l'ancestralité dans sa dimension classique dans des groupes sociaux habituellement pensés comme dans la perte de ses filiations en raison du régime esclavocrate qui les ont constitués. Cette opinion courante, d'absence de ruines et/ou d'ancêtres a été largement relayée par les poètes et écrivains antillais. Pareillement les 
anthropologues ${ }^{4}$ ont souvent conclu à un non-savoir généalogique dû à l'absence de territorialisation des ancêtres, tout en notant cependant l'existence de mauvais morts dont l'action maléfique rejaillit sur les vivants.

La vague patrimoniale qui balaie depuis la fin des années 1980 l'ensemble de la planète ne touche pas uniquement des groupes en quête identitaire, elle gagne également des institutions, et les plus fréquemment happées par une vague mémorielle sont les États postcoloniaux en plein processus de démocratisation avec les conflits qui les accompagnent. Pour créer une intersubjectivité trans-ethnique à caractère national, la qualité d'ancêtre peut prendre la forme complète du corps d'un humain ou d'un animal. Il peut aussi évoquer seulement une des partie du corps (les os), des métaphores et/ou métamorphoses (mausolée, cercueil, animal, procès-verbaux, chants et champs).

Toutes ces thèses interrogent la mémoire dans son aspect instrumentalisé qu'il convient de dépasser. L'ensemble des articles présentés dans ce dossier sur l'Ancestralité revisitée se développe autour de trois axes :

\section{Le premier interroge les processus d'ancestralisation à l'ouvre dans la construction de l'État-nation}

Autour de cet axe sont mis en lumière les processus d'ancestralisation visant à former et consolider l'État-nation et/ou qui accompagnent des mobilisations autour de la question de la formation de l'État-nation. L'esclavage, la colonisation, les conflits armés et la démocratisation des sociétés participent des contextes sociohistoriques dans lesquels émergent des figures ancestrales loin de tout agencement familial.

Bruce Berman et John Lonsdale (1992) distinguent la formation de l'État comme institution sociale, de sa construction comme appareil administratif. Ils soulignent que le raisonnement en termes de construction de l'État tend à réduire l'histoire des États à leur période fondatrice et aux actions des agents officiels. Ils proposent, par contre, une approche en termes de formation qui a le mérite d'appréhender les trajectoires étatiques dans la longue durée, tout en intégrant le jeu des acteurs privés. Cette variation de point de vue permet à ces auteurs de montrer que les sociétés s'invitent systématiquement dans le processus de formation de l'État à travers leurs techniques de « vulgarisation du pouvoir ", qui consistent en l'appropriation de l'autorité publique à des fins privées. Cette approche présente des vertus heuristiques en ce qu'elle permet de cerner comment les mobilisations sociales autour de certaines figures pour les ancestraliser, participent de la formation de l'État.

Ainsi, au Congo-Brazzaville, la célébration du $126^{\text {ème }}$ anniversaire de la capitale du pays autour de Pierre Savorgnan de Brazza, figure de l'ordre républicain colonial, sert de prétexte à Rémy Bazenguissa-Ganga pour examiner les figures qui participent à la formation d'un nouvel ordre politique. S'attachant à une histoire de moyenne durée, il interroge les différentes controverses suscitées par la construction d'un mausolée en mémoire de l'ancien colonisateur. Du premier traité entre de Brazza et le roi Teke, rejouant la scène des gens du pouvoir et des gens de la terre, pour acter la naissance et l'origine de l'État-nation contemporain, Rémy Bazenguissa-Ganga questionne également le fait de la transformation du colonisateur en ancêtre fondateur 
de l'État-nation congolais sans que la mémoire du roi Teke, le Makoko ne soit célébrée dans le même temps. Le rapatriement de ses os de l'Algérie où il avait été enterré est l'occasion de s'interroger sur les os d'autres disparus, en considérant les uns et les autres comme les os de la république. Rémy Bazenguissa-Ganga égrène une série d'événements dans lesquels la mort et la dissolution du corps vivant en os appelle une analyse sociopolitique de l'État congolais : ainsi, il rappelle que si tous les morts ne sont pas égaux, il y en a qui le sont plus que d'autres, en ce que leurs os ou leur absence peut les ériger en messie dont on attend patiemment le retour, comme c'est le cas pour André Matswa, héros malheureux de l'anticolonialisme. Pour d'autres, au contraire, la mort et la dissolution du corps et des chairs jusqu'à ce qu'il ne reste que les os ne fait l'objet d'aucun traitement spécial, comme pour tous ces morts anonymes victimes des guerres entre 1990 et 2000 dans le pays. Rémy Bazenguissa-Ganga constate que dans le contexte de son étude, les processus d'ancestralisation sont ordonnés selon des paradigmes qui s'entrecroisent, ceux du sang associé à la terre dans un premier cas et ceux de l'écriture associée à la déshérence dans le second cas. D'un côté la filiation et la territorialisation, de l'autre, le pouvoir scripturaire des orphelins.

Si l'article de Rémy Bazenguissa-Ganga concerne une figure humaine qui va être ancestralisée pour consolider un pouvoir postcolonial affaibli par les nombreux conflits qui ont accompagné à partir des années 1990, les processus de démocratisation du pays, à Madagascar, le cas analysé par Thomas Mouzard concerne la transformation d'une figure animale en figure ancestrale razamasy, « ancêtre-sacré » dont les traits au cours de son périple de trois mois à travers le territoire malgache vont être appropriés et transformés à chaque étape, jusqu'à sa dernière transformation en sirène. L'action se déroule entre le 21 octobre 2001 et le 21 janvier 2002 avec la procession d'un cercueil qui traverse le Sud du pays d'Est en Ouest depuis les environs de Tôlanaro (FortDauphin) jusqu'à Toliara (Tuléar). Mouzard qui est présent à Tuléar au moment des faits prend connaissance de cette étrange procession à travers la rumeur selon laquelle, à Fort-Dauphin, un pêcheur aurait attrapé une sirène dans ses filets et l'aurait ramenée au village. Là, la sirène finit tuée par des hommes effrayés par sa capacité à boire le rhum sans manifester la moindre ivresse. Mais avant de mourir, elle maudit ses assassins et exige d'être enterrée dans le village d'Ankilibe près de Tuléar, où elle devra être conduite par des porteurs qui se relaieront de village en village jusqu'à sa destination finale. Et ceux qui voudront lui demander des bénédictions devront le faire en posant la main droite sur son cercueil. En remontant les différentes étapes, relais suivis par le cercueil jusqu'à son point de départ, Mouzard recueille les différents éléments qui vont s'agréger pour transformer un lémurien (aye-aye), en figure ancestrale dont les bénédictions sont très recherchées comme en témoignent les nombreux procès-verbaux qui ont accompagné son passage de village en village.

Dans le cas du Brésil, c'est dans un culte de possession que l'on retrouve l'imaginaire national d'une société bâtie par des colons qui ont en grande partie éradiqué les autochtones tout en les transformant en ancêtres. Kadya Tall montre ainsi que l'africanité revendiquée des maisons de candomblé bahianais, loin de participer de l'imaginaire d'une Afrique en conserve, s'articule à l'idéologie de la fraternité raciale dans la mise en présence réelle, lors des rituels, de différentes catégories de figures évoquant la société coloniale. La description d'un rituel dédié aux ancêtres d'une maison de candomblé et de sa communauté qui s'identifient comme appartenant à la nation 
Ketu, en référence à l'ancien royaume de Kétou situé dans l'actuel Bénin, permet de comprendre que l'espace-temps dans lequel s'inscrit la célébration des ancêtres, ainsi que leur diversité désigne une Afrique bien particulière, celle de l'espace atlantique du commerce négrier, de la contre-réforme catholique et de la colonisation des terres du Nouveau monde. Cet espace-temps est saisissable dans la date choisie pour célébrer les morts et les ancêtres de la maison - la nuit du $1^{\text {er }}$ au 2 novembre - la qualité des offrandes et des mets consommés lors de cette veillée et les espaces différenciés dans lesquels se déroule la célébration, et surtout la multiplicité des ancêtres évoqués, formant ainsi une mosaïque de nations. Ainsi, contrairement à la doxa qui insiste sur le caractère africain du rite du candomblé, en particulier celui de nation Ketu, dès lors que l'on examine les pratiques et les liens généalogiques réels et religieux du personnel liturgique, la référence ketu s'estompe au profit des autres nations africaines, européennes et amérindiennes. Enfin, si la célébration des ancêtres divins et humains vise à réactiver les liens entre ascendants et descendants humains et divins, cette commémoration au sein d'une maison de candomblé vise également à inscrire la diversité et la pluralité des origines dans l'imaginaire communautaire.

L'article de Christine Hardung se situe à la frontière du premier axe, et du second qui concerne les territoires de l'ancestralité. En effet, les recherches historiques menées par cette auteure sur Hendrik Witbooi, figure de la résistance à la domination coloniale dans le Sud-Ouest de l'Afrique du Sud du $19^{\text {ème }}$ siècle, la conduise à considérer la croisade/expédition/campagne de cette figure prophétique comme un moyen de légitimer un droit sur un territoire.

L'ambiguïté de cette figure réside dans sa profonde adhésion à la foi chrétienne et son recours aux Ancêtres pour légitimer la quête territoriale du groupe Nama-Oorlam qui rassemblait alors des déserteurs, des esclaves fugitifs et des Khoekhoe ayant été expropriés de leurs terres de pâture. Pratiquant le raid du bétail, ces groupes et leur leader forment ce que l'auteure désigne comme une " communauté charismatique de violence " . Witbooi, le chef de guerre et prophète va instaurer des règles strictes à ses troupes afin qu'ils internalisent leur foi. Ce dernier use des techniques habituelles dans les pratiques religieuses (vision, jeûne, abstinence sexuelle, etc.), toutes techniques de domestication du corps pour le rendre pur et activer les puissances qui l'habitent. Dans le cas de Witbooi, il s'agit de Dieu et des Ancêtres. La révélation divine et la conversation chemin faisant avec les ancêtres vont lui permettre de re-tracer le chemin du passé pour le futur sur le territoire de l'ancien frère ennemi avec qui l'on se réconcilie pour former un nouvel État. Territoire de nomade qui s'étend de part et d'autre du chemin à suivre. La conversion au Christianisme semble être ici un outil permettant de re-trouver le chemin des ancêtres.

\section{Les territoires de l'ancestralité}

Le second axe aborde l'intervention, pour préserver l'intégrité d'un territoire, de certains dispositifs «performatifs » de subjectivation de l'ancêtre : la nomination, la diasporisation ou consolidation de l'espace public (société civile) contemporain, la séparation de la nature et la composition de chants.

Aux Antilles, Christine Chivallon propose de mettre en regard ses récents travaux sur une mémoire patronymique d'une insurrection survenue en 1870 avec 
celle d'une mémoire ancestrale inventée. S'attachant à la dimension généalogique de l'ancestralité, elle propose d'examiner les quatre paliers successifs qui lui permettent d'appréhender l'ancestralité aux Antilles et à la Martinique : le premier est le processus de nomination des ancêtres, le second palier est la description d'une insurrection paysanne qui va révéler la puissance d'un nom patronymique et le troisième ramène l'auteure à questionner l'attribution d'un patronyme au seul colon dominateur et enfin pour conclure Christine Chivallon propose une mise en miroir des pratiques récentes de recomposition ancestrale avec les agencements familiaux examinés. Les interrogeant dans leur aspect phénoménologique d'expérience du sensible à travers le souvenir, Chivallon s'est attachée à des faits du passé marquants dont le plus ancien est l'insurrection paysanne plus connue sous l'affaire Codé, survenue en 1870, soit une vingtaine d'années après l'abolition de l'esclavage en 1848. Insurrection d'une petite paysannerie noire suffoquée par la domination des planteurs blancs malgré l'instauration d'une Troisième République, mais surtout insurrection qui sera transformée en vulgaire affaire criminelle où d'un côté, il y avait d'horribles sauvages sanguinaires noirs et de l'autre une pauvre victime, le planteur blanc assassiné lors de cette révolte, encore aujourd'hui considéré comme le héros qui s'est sacrifié pour l'honneur et la grandeur de la République française. Conduisant certains descendants d'insurgés à taire le patronyme honteux par crainte de la malédiction du mort assassiné et/ou de l'opprobre générale et à d'autres à se disperser et à abandonner leurs terres. Honte et crainte du mort mêlées ont conduit à des changements patronymiques et à la dispersion de la famille et la perte des terres selon les interlocutrices de l'auteure, mais ces changements loin de forger l'oubli, au contraire ranime le souvenir tant chez les autres qu'au sein de la famille meurtrie par la honte et le déshonneur. Cette perte du nom et des terres permet à Christine Chivallon de parler de territoires patronymiques en ce que « Tout au long des récits, la centralité occupée par la référence à ce qu'il a paru opportun d'appeler des ‘territoires patronymiques'» (Chivallon 2012 : entre autres 282, 407) vient confirmer que l'institution première de la culture « contre-hégémonique $»^{5}$, à la Martinique, réside bien dans l'entité famille-patrimoine de la paysannerie fondée par les anciens esclaves. En découvrant des territoires patronymiques qui tout à la fois dessinent des territoires et des généalogies agnatiques et non utérines, Chivallon bouscule les idées reçues sur la matrifocalité aux Antilles.

Si dans le cas Codé, les territoires ruraux deviennent des territoires patronymiques pour des descendants d'esclaves en Martinique, le contexte chinois analysé par Anne-Christine Trémon apparaît comme un contrepoint intéressant, dans la mesure où là, des ancêtres villageois se transforment en ancêtres de la société civile avec l'expansion accélérée d'une petite commune, Fort-Les-Pins, en zone urbaine et industrielle. La présence des anciens lignages et la persistance d'une forme d'économie collective contrebalancent le pouvoir de l'État et le culte des ancêtres qui avait été banni lors de la Révolution culturelle et exporté à Hong Kong par des membres de la diaspora qui en avait poursuivi le rite en remplaçant la tablette jetée à la mer. Cependant depuis la fin des années soixante-dix et l'ère dite des « réformes et de l'ouverture », les activités cultuelles et religieuses ont repris en Chine notamment dans les années 1980-

5 Selon l'expression empruntée à Baron et Cara (2003: 5) pour définir les pratiques défiant la domination culturelle dans les mondes créoles issus de la matrice esclavagiste. 
1990. Ainsi à Fort-Les Pins, en 1981, la tombe de l'ancêtre Zhenneng avait été rénovée, la tablette repêchée avant d'être remplacée quelques années plus tard, grâce aux deniers des Chen de Hong Kong et d'outre-mer. Trémon observe, dans cette revitalisation des cultes, un contraste entre l'individualisme du secteur capitaliste privé et une économie collective qui soutient la recherche du bien public. Ses recherches sur les liens entre un lignage local et sa diaspora montrent que tous les dons de cette dernière sont toujours localement présentés comme une contribution au bien public. Trémon précise qu'elle s'intéresse plus particulièrement au statut ontologique de l'ancêtre fondateur de lignage qui a très peu été étudié en Chine en cherchant à prolonger les travaux de Maurice Freeman qui avait déjà proposé une réflexion sur la tension entre culte individuel des ancêtres immédiats et culte collectif de l'ancêtre commun. Trémon va ainsi montrer comment le statut de cette figure à la croisée du privé et du collectif s'est transformé pour se partager aujourd'hui entre individuel et public.

Dans le texte de Magda Helena Dziubinska, une fois encore, c'est pour la préservation d'une identité territoriale, que les Kakataibo d'Amazonie péruvienne reconstruisent leur rapport à l'Autre qui, de sauvage et brutal, devient fragile et à protéger. Mais la particularité de cet Autre dangereux est également dans le fait que, chez les Kakataibo comme dans de nombreuses sociétés amazoniennes, la figure de l'Ancêtre est généralement considérée comme un ennemi prédateur. Comment s'opère le basculement d'une vision honnie de l'ancêtre en figure bienveillante à protéger des dangers de la modernité ? Dziubinska voit dans ce retournement singulier, la problématique identitaire propre aux Amérindiens ayant perdu les signes diacritiques qui les différencient de la société nationale. La figure de l'altérité que cherche à protéger les Kakataibo dés-indianisés est celle du Kamano, un être dont la pâleur, la ressemblance avec les Européens (haute taille et rondeurs) et le langage est difficile à comprendre. Jusqu'à peu, on ne cherchait pas à les amadouer pour en obtenir des faveurs. Dziubinska souligne que la confusion faite entre Indiens non contactés, esprits de la forêt et narcotrafiquants réfugiés en forêt est fréquente chez les Indiens vivant en lisière de la forêt amazonienne. En tant qu'être malfaisant et violent, le Kanamo est assimilé à la figure de l'ancêtre pilleur et voleur et il est intéressant de comprendre comment le discours des ONG écologiques et des organismes publics de protection des groupes amérindiens va transformer cette figure négative en figure positive pour les jeunes générations Kakataibo. En effet, si l'emprunt aux groupes voisins est une constante en Amazonie, le renversement radical de la perception de l'ancêtre doit être contextualisé. En effet, si à l'étranger les Kakataibo sont considérés comme de vrais indiens, il en est différemment au Pérou où leur longue immersion dans la société globale est considérée comme une perte de traditions, notamment chamaniques, signe diacritique d'autant plus important qu'un groupe voisin, les Shipibo-Conibo sont des spécialistes reconnus $\mathrm{du}$ chamanisme avec ingestion d'ayahuasca, la liane hallucinogène. Cependant en positivant leurs relations avec les Kanamo, ces Amérindiens en isolement, et en devenant leur garant vis-à-vis de la société globale, les kakataibo ont re-trouvé leur identité amérindienne, en devenant comme d'autres groupes amazoniens, les garants de la préservation de la forêt et des savoirs endogènes. On ne saura pas si les Kanamo sont des créatures humaines ou des inventions issues de l'esprit des Kakataibo mais on apprend que grâce à leur affiliation généalogique avec les premiers, ces derniers sont devenus performants sur le marché ethnique. 
La réappropriation de territoires géographiques et/ou symboliques n'est pas l'apanage des sociétés issues de l'esclavage et la vague patrimoniale qui qualifie le présentisme comme régime d'historicité a remis à l'honneur dans de nombreux pays africains les Autorités précoloniales.

Jean-Baptiste Manga étudie la royauté d'Oussouye et c'est dans le cadre de ses recherches sur le retour de la royauté sur la scène politique sénégalaise qu'il s'est intéressé aux chants dédiés aux ancêtres. Un même terme, Kuhuluy désigne un autel, un interdit d'évocation de nom de défunt et le répertoire de chants qui accompagne la célébration d'un ancêtre. Si l'influence des religions révélées a un temps fait passer la célébration des ancêtres au second plan, depuis quelques années, avec la migration nationale et transnationale, le retour des ancêtres permet de consolider les liens, de revitaliser une identité socio-ethnique et d'une manière générale, de se situer pour les nouvelles générations soucieuses de conserver leur patrimoine. En décrivant et analysant les différents éléments qui composent la célébration des ancêtres sur le territoire royal d'Oussouye, Manga éclaire la complexité des relations de genre dans une société où en dépit d'un penchant plutôt patrilinéaire, on voit, notamment dans la création des chants funéraires, l'importance des femmes qui tissent les chants comme autrefois elles tissaient le pagne $(k a h u l)$ qui couvre le mort dans sa dernière demeure. L'importance des chants réside dans le fait qu'une fois mort, le défunt ne peut plus être évoqué par son nom de vivant et que c'est à travers le chant que sa mémoire va être conservée dans le groupe. Le chant va donc essentiellement consister en la création d'un surnom et de louanges. Les femmes créent les chants au cours de soirées pendant lesquelles elles se réunissent en famille, en général les agnates du défunt, mais ce sont des hommes qui en conserveront la mémoire et lanceront les chants lors des veillées funéraires. En principe chaque quartier a son chantre (atika) mais ils peuvent être plusieurs à se succéder à tour de rôle lors des veillées. L'apprentissage des chants se fait collectivement au cours des rites de clôture du deuil et dès lors, le ou la défunte sera évoqué par ses chants à toutes occasions qu'elles soient formelles ou informelles. Cependant si au village, les occasions ne manquent pas pour transmettre les chants, en milieu urbain et en contexte transnational, la chaîne de transmission peut se briser et le recours aux techniques audiovisuelles devient le moyen privilégié pour l'éviter.

\section{Ancestralité et Filiation}

Dans ce troisième et dernier axe, la consanguinité semble resurgir en l'état, comme dans l'anthropologie classique où l'ancêtre semblait déterminer un ordre juridique et l'autorité des anciens. Mais ne nous y trompons pas car en effet, les ancêtres sont plutôt ici des productions individuelles. En effet, si l'imaginaire amérindien semble (re)construire une généalogie dans une société habituellement considérée comme sans ancêtres ou avec une mémoire ancestrale maximale à deux générations, ce sont des urbains qui, en mobilisant ces figures, négocient leur place sociale dans le village quand cela est nécessaire. Par ailleurs l'intrusion des avancées technologiques notamment dans la science génomique, semble réinscrire la consanguinité dans les recherches de filiation et d'ancêtres. On verra pourtant que ces recherches, loin de définir un champ scientifique de certitude s'apparente largement à des imaginaires sociaux dans lesquels la race, redevient un primat. 
Cédric Yvinec questionne certaines idées reçues sur l'absence des ancêtres dans les jeux sociaux des Amérindiens d'Amazonie. Menant des recherches sur les Suruí du Rondônia, groupe qui a eu ses premiers contacts avec la société brésilienne à la fin des années 1960, Yvinec rappelle que les sociétés amazoniennes se caractérisent par une faible profondeur généalogique, deux générations au plus, impliquant l'absence de lignées transmettant des droits ou des biens. Mais chez les Suruí du Rondônia comme chez les Kakataibo du Pérou, les morts sont considérés comme des prédateurs de vivants et les rituels funéraires visent à oblitérer leur souvenir. Assimilés à des affins plutôt qu'à des ascendants, les morts dont la mémoire est gardée sont sollicités pour chasser les spectres des morts récents et c'est en général l'unique fonction qui leur est attribuée dans les pratiques funéraires. Ainsi, les ancêtres comme collectif garant de l'ordre social n'existent pas en Amazonie, cependant, l'appropriation individuelle de certains morts n'est pas rare dans cette région, même si celle-ci fait l'objet de peu de ritualisation. Yvinec montre de manière fort convaincante combien, ces appropriations individuelles d'ancêtres permettent de résoudre à son avantage des enjeux matrimoniaux et politiques, en particulier dans les relations qu'ils instaurent entre père et fils, ainsi qu'entre frères et entre beaux-frères dans une société où « la seule règle de mariage est que les oncles maternels réels ont tous les droits sur leurs nièces utérines - y compris d'en accaparer une demi-douzaine », provoquant inéluctablement des inégalités et un célibat long pour les jeunes hommes.

Si l'ancêtre comme collectif auquel on peut se référer n'existe pas chez les Suruí, il existe cependant une théorie eschatologique dans laquelle le chemin du mort est semé d'embûches que les plus faibles qui ne les surmontent pas se voient transformer en termites tandis que les morts ayant vaincu dans l'épreuve deviennent des êtres craints par les vivants. Pour vaincre, il faut avoir mené une vie exemplaire (interdit de l'inceste, du meurtre d'un Suruí, maîtrise des arts de la chasse et avoir tué des ennemis en grand nombre), chose nous dit l'auteur atteignable par la majorité des Suruí, car ces valeurs participent au prestige politique et matrimonial et ceux qui n'y parviennent pas sont bien souvent des célibataires. Ces morts devenus puissants se transforment en ancêtres protecteurs grâce aux rêves que les vivants font d'eux. Rêver d'un mort est la preuve de sa protection. Il est alors désigné comme ascendant et comme grand-père agnatique et sa protection est une arme contre les parents utérins, c'est-à-dire les oncles maternels. Toutefois, le récit de cette rencontre ne se fait pas publiquement, une certaine discrétion doit être assurée dans la mesure où la protection d'un ancêtre se fait toujours au détriment des autres parents qui pourraient s'en revendiquer et que son action vise à annihiler les actions néfastes des proches. Dans l'étude de cas présentée par l'auteur, on voit comment la rencontre en rêve d'un individu avec son grand-père et le récit qu'il en fait à son père, récit que ce dernier accepte comme vrai, lui permet de renégocier sa place au sein de sa famille, notamment vis-à-vis de ses frères qu'il juge recevoir plus de compassion de leur père que lui-même. En racontant son rêve à l'auteur, après l'avoir raconté à sa femme et à la mère de celle-ci, il espère que la rumeur va se propager et accroître sa légitimité au sein de son groupe.

Rêver pour construire le lien avec un ancêtre, jamais trop lointain, permet donc chez les Suruí de se protéger et d'augmenter par là sa puissance et sa légitimité au sein du groupe. À l'inverse dans nos sociétés biotechnologiques, le lien généalogique peut acquérir une profondeur abyssale grâce aux recherches en génétique. 
Comme le remarque Chimamanda Ngozi Adichie dans son dernier roman Americanah, on a beau seriner que la race n'existe pas scientifiquement, que c'est une construction sociale, etc., tant dans le domaine de la santé et de la prévention des maladies " héréditaires » que dans la quête identitaire des populations du Nouveau monde, la question des origines et sa racialisation sont très prégnantes et s'en extraire peut être considéré comme un déni, un délit de fuite, une illusion aussi nocive que celle qui consiste à trouver des fondements naturels à cette construction sociale.

Les anthropologue et éco-anthropologue Jean-Luc Bonniol et Pierre Darlu interrogent ainsi dans leur article l'impact des technologies, en particulier le séquençage génétique dans la demande sociale. Notant combien la question des origines devient une obsession récurrente dans nos sociétés postindustrielles, ces auteurs soulignent combien les avancées du savoir biologique ont favorisé une appréhension renouvelée de la diversité humaine, faisant resurgir les théories raciales du $19^{\text {ème }}$ siècle. Et ce, en dépit des travaux des généticiens qui s'emploient à les démystifier, en rappelant que l'être humain est génétiquement semblable à son voisin pour 99,9\% de ses gènes. Les $0,1 \%$ restants qui pourraient être comme pour le whisky, la part des Anges, est au contraire l'objet de multiples interprétations qui ne sont pas exemptes d'illusions et de constructions raciales. Particulièrement présentes sur internet, dans les offres de tests génétiques pour vérifier sa paternité ou découvrir ses ancêtres, nos auteurs notent que « l'ADN peut être considéré comme une trace des origines, ou une incarnation des ancêtres, qui implique tout à la fois la représentation de sa généalogie (et donc de son hérédité...) et la perception d'un éventuel risque hérité touchant à la santé ».

Ainsi le succès commercial des sites internet proposant des tests génétiques pour compléter des recherches généalogiques, connaître son pourcentage de race européenne, africaine, asiatique ou amérindienne sont florès et alimentent la racialisation de l'espèce humaine pourtant mise à mal par les résultats de la science génétique. Le paradoxe serait moindre si certains de ces sites n'étaient pas dirigés par d'éminents scientifiques comme Y. Brian Sykes ou encore par des intellectuels comme Henry Louis Gates Jr.

On conclura par une note positive, en rappelant que si l'illusion continue d'être la maîtresse qui guide les quêtes identitaires de nos contemporains, il faut bien évidemment que la fiction colle à la biographie de chacun, et plus qu'avant, au-delà des constructions raciales, c'est la représentation que chacun a de soi qui permet de faire des choix.

Revisiter l'ancestralité permet donc de questionner notre présent historique aussi bien du point de vue social qu'individuel. Les formes promues par ces nouvelles pratiques d'ancestralisation interviennent dans les processus de formation et consolidation de l'État-nation dans le cadre de la mondialisation. L'esclavage, la colonisation, les conflits armés et la démocratisation des sociétés dessinent là des répertoires sociohistoriques d'où émergent des figures ancestrales loin de tout agencement familial. La nomination d'un ancêtre n'est plus seulement le fait d'une communauté, d'un groupe social et l'individu peut également intervenir fortement dans cet ordre. Ces processus s'accompagnent aussi de dispositifs qui en les nommant, font les ancêtres. L'ordre du temps, le régime d'historicité, se trouve ainsi inversé, l'ancêtre ne représente plus seulement le passé qui n'en finit pas de mourir, mais reste un produit du présent. Plus encore, à travers lui le passé est à produire continuellement. Les ancêtres atteignent ainsi au pouvoir de représentation dont Louis Marin (1986) souligne que c'est le principe de nomination qui 
l'active. Nommer des ancêtres c'est (re)donner vie à une autorité qui vient légitimer des droits pour les acteurs sociaux.

\section{Références citées}

Adichie, Chimamanda Ngozi, 2015. Americanah. Paris : Gallimard.

BARON, Robert et Ana CARA, 2003. "Introduction : Creolization and Folklore - Cultural Creativity in Process », Journal of American Folklore, 116 (459), pp. 4-8.

Berman, Bruce et John Lonsdale, 1992. Unhappy Valley : Conflict in Kenya and Africa. Londres : James Currey.

Bougerol, Christiane, 1987. «L'involontaire retour des morts aux Antilles », Etudes rurales, 105-106, pp. $65-78$.

Chivallon, Christine, 2012. L'esclavage, du souvenir à la mémoire. Contribution à une anthropologie de la Caraïbe. Paris : Karthala.

DE Witte Marleen, 2001. Long Live the Dead! Changing Funeral Celebrations in Asante, Ghana. Amsterdam : Aksant Academic Publishers.

ForTES, Meyer, 1965. « Some Reflections on Ancestors Worship in Africa », in Fortes et Dieterlen, African System of Thought, pp. 122-144. Londres : Oxford University Press.

Gluckman, Max, 1937. "Mortuary, Customs and the Beliefs in Survival after Death among the SouthEastern Bantu », Bantu Studies, xi.

Goody Jack, 1962. Death, Property and the Ancestors. A study of the Mortuary Customs of the Lodagaa of West Africa. Stanford : Stanford University Press.

Hartog, François, 2003. Régimes d'historicité. Présentisme et expériences du temps. Paris : Seuil.

Hertz, Robert, 1970 [1907]. "Contribution à une étude sur le représentation collective de la mort », Sociologie religieuse et folklore, pp. 1-83. Paris : PUF.

JINDRA, Michael, 2005. « Christianity and the Proliferation of Ancestors : Change in Hierarchy and Mortuary Ritual in the Cameroon Grassfields », Africa, 75 (3), pp. 356-377.

Kopytoff, Igor, 1971. « Ancestors as Elders in Africa », Africa, 41 (2), pp. 129-142.

KuPER, Adam, 1982. « Lineage Theory : A Critical Retrospect», Annuel Reviews, 11, pp. 71-95.

LeIrIS, Michel, 1987 [1955]. Contacts de civilisation en Martinique et en Guadeloupe. Paris : Unesco/ Gallimard.

MARIN, Louis, 1986. La parole mangée et autres essais théologico-politiques. Paris : Méridiens Klincksieck.

McCALL, John. 1995. Rethinking Ancestor in Africa, Africa, 65 (2), pp. 256-270.

Noret, Joël, 2010. Deuil et funérailles dans le Bénin méridional. Enterrer à tout prix. Bruxelles : Éditions de l'Université de Bruxelles.

Systèmes de Pensée en Afrique noire, 1989. Cahier 9.

-, 1991. Cahier 11.

- , 1994. Cahier 13.

Tonda, Joseph, 2000. «Enjeux du deuil et négociation des rapports sociaux de sexe au Congo », Cahiers d'Études africaines, 157, pp. 5-24.

Vidal, Claudine, 1986. « Funérailles et conflit social en Côte d’Ivoire », Politique africaine, 24, pp. 9-19. 